\title{
Creation of Long-Term Coherent Optical Memory via Controlled Nonlinear Interactions in Bose-Einstein Condensates
}

\author{
Rui Zhang, Sean R. Garner, and Lene Vestergaard Hau \\ Department of Physics and School of Engineering and Applied Sciences, Harvard University, Cambridge, Massachusetts 02138, USA
}

(Received 16 September 2009; published 4 December 2009)

\begin{abstract}
A Bose-Einstein condensate confined in an optical dipole trap is used to generate long-term coherent memory for light, and storage times of more than $1 \mathrm{~s}$ are observed. Phase coherence of the condensate as well as controlled manipulations of elastic and inelastic atomic scattering processes are utilized to increase the storage fidelity by several orders of magnitude over previous schemes. The results have important applications for creation of long-distance quantum networks and for generation of entangled states of light and matter.
\end{abstract}

DOI: 10.1103/PhysRevLett.103.233602

PACS numbers: 42.50.Gy, 03.75.Mn, 03.75.Nt, 32.80.Qk

Quantum information science is a very active area of research, and the quest to create long-distance quantum networks is at the heart of current activities. Such networks would allow for quantum key distribution and teleportation over long distances, and for quantum computing with distributed processors [1]. Since light is an efficient carrier of both classical and quantum information, typical network designs rely on optical interconnects that link matter-based nodes where processing can take place. Therefore, methods for reversible transfer of information between light and matter are required, and efficient and coherent mapping of optical states to and from an atomic medium is possible with the use of ultraslow light in cold atom clouds under conditions of dark states and electromagnetically induced transparency [2]. Light storage and revival has been demonstrated for light pulses with classical statistics [3,4], for single photon pulses [5,6], and for entangled states of light with the entanglement preservation determined entirely by the fidelity for classical light storage [7]. Storage times of a few milliseconds were obtained for both classical light $[3,4]$ and single photon pulses [8,9], but for the development of long-distance quantum networks longer storage times are desirable $[10,11]$.

The storage time in atomic ensembles is limited mainly by thermal diffusion and by loss of atomic coherence due to collisions, and one way to suppress these effects is to keep the atoms localized. This philosophy was used for storage of classical light pulses in rare-earth doped insulators cooled to a few Kelvin $[12,13]$ and in a Mott insulator with cold atoms in an optical lattice [14]. In the former case, storage times in the second regime were obtained [13] but the experiments relied on a spin-echo technique with use of rf rephasing and extension of the method to the quantum regime remains challenging [15]. In the latter case, light pulses were stored for $600 \mathrm{~ms}$ but with a fidelity of only $0.03 \%$ [14].

Here, we use slow light in Bose-Einstein condensates (BECs) which represent a state of matter where thermal diffusion is completely suppressed. We map an optical

pulse onto the condensate wave function, creating a localized matter wave imprint in the BEC. We manipulate the nonlinear properties of the condensate by controlling both the real and imaginary parts of the atomic scattering lengths governing interactions between the imprint and the condensate. Inelastic collisions are minimized, and a phase-separating regime is entered whereby the imprint is protected over large time scales in a self-induced void in the BEC. The imprint is also controllably positioned to minimize losses during regeneration and read out of the optical pulse.

In the experiments, sodium atoms in a condensate are illuminated by coupling and probe laser fields that couple three internal levels as shown in Fig. 1(a). The coupling beam is resonant with the $|2\rangle \rightarrow|3\rangle$ transition, and when the probe beam is tuned on resonance with the $|1\rangle \rightarrow|3\rangle$ transition, a dark state is formed where an atom is in a coherent superposition of states $|1\rangle$ and $|2\rangle$ [16]:

(a)

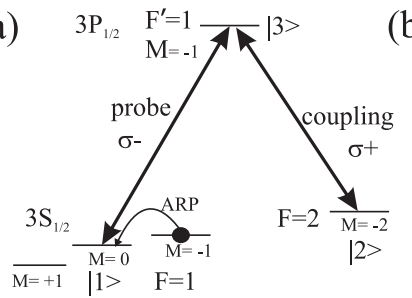

b) $\hat{\mathrm{y}}$

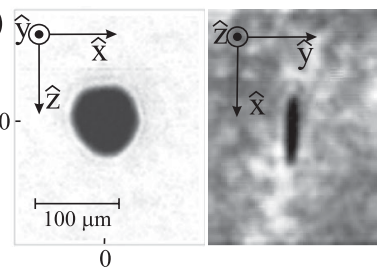

(c) probe

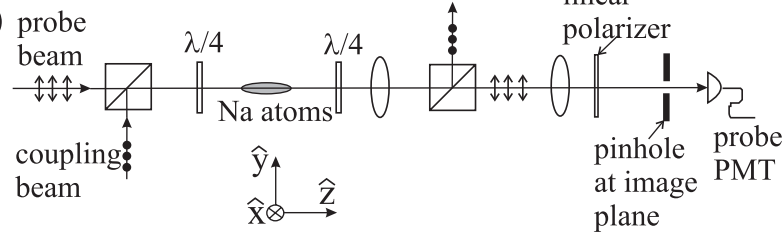

FIG. 1. (a) The internal quantum states for sodium atoms used in our experiment. (b) Shadow images of a BEC in the optical dipole trap. (c) Schematic drawing of the experimental setup. 


$$
\begin{aligned}
& \Psi_{D}(\mathbf{R}, t)=\psi_{1}(\mathbf{R}, t)|1\rangle+\psi_{2}(\mathbf{R}, t)|2\rangle, \\
& \frac{\psi_{2}(\mathbf{R}, t)}{\psi_{1}(\mathbf{R}, t)}=-\frac{\Omega_{p}(\mathbf{R}, t)}{\Omega_{c}(\mathbf{R}, t)} \exp \left[i\left(\mathbf{k}_{p}-\mathbf{k}_{c}\right) \mathbf{R}-i\left(\omega_{p}-\omega_{c}\right) t\right] .
\end{aligned}
$$

Here $\Omega_{c}\left(\Omega_{p}\right), \mathbf{k}_{c}\left(\mathbf{k}_{p}\right)$, and $\omega_{c}\left(\omega_{p}\right)$ are the Rabi frequency, the wave vector, and the optical angular frequency of the coupling (probe) laser, respectively, and $\psi_{1}$ and $\psi_{2}$ are matter fields for atoms in $|1\rangle$ and $|2\rangle$. [The probe laser field and $\psi_{2}$ may be represented by field operators (relevant for few photon pulses and quantum states) whereas the coupling laser field and the $\psi_{1}$ condensate component are treated as mean fields.] In the dark state, excitation amplitudes to state $|3\rangle$ induced by the two laser beams destructively interfere and neither probe nor coupling beams are absorbed. The quantum interference also leads to a rapid variation of the refractive index for the probe laser field as a function of its detuning from the $|1\rangle \rightarrow|3\rangle$ resonance.

Initially, atoms are in state $|1\rangle$ and illuminated by the coupling laser only. A probe pulse is then injected and the large slope of the refractive index causes a dramatic deceleration and associated spatial compression of the light pulse (by a factor of $3 \times 10^{7}$ for our experimental parameters), and within the localized probe pulse region atoms are in the superposition state of Eq. (1) [2]. The probe laser field is thus mapped onto the atom wave function (shared by all atoms in the condensate), forming an "imprint" $\left(\psi_{2}\right)$ of the probe laser pulse's amplitude and phase that coincides with the probe pulse in space and time. In a dense atom cloud, such as a BEC, pulses of microseconds duration can be entirely contained, and a subsequent turn off of the coupling laser leads to extinction of the probe light pulse with its imprint stored in the atom cloud. When the coupling beam is turned back on, the procedure reverses and the probe pulse is regenerated [3].

To trap atoms in both $|1\rangle$ and $|2\rangle$, we load a sodium BEC with $3 \times 10^{6}$ atoms into an optical dipole trap [17] created by two crossed, far-detuned laser beams propagating in the $x$ and $z$ directions (Fig. 1). Each trapping beam has a power of $500 \mathrm{~mW}$ and a wavelength of $980 \mathrm{~nm}$. This results in a pancake-shaped BEC with a diameter of $80 \mu \mathrm{m}$ in the $x$ and $z$ directions and a thickness of $15 \mu \mathrm{m}$ in the $y$ direction [Fig. 1(b)]. The condensate is formed with atoms in $|3 S, F=1, M=-1\rangle$, and using adiabatic rapid passage (ARP) [18], we apply an rf field to transfer all atoms to state $|3 S, F=1, M=0\rangle$, which is state $|1\rangle$ for our experiments [Fig. 1(a)]. A bias magnetic field, $B=18 \mathrm{G}$, in the $z$ direction is present during the ARP and nonlinear Zeeman shifts are used to control the population transfer.

According to our calculations based on Ref. [19], the imaginary part of the $s$-wave scattering length $\operatorname{Im}\left(a_{12}\right)$ (governing losses in collisions between atoms in $|1\rangle$ and $|2\rangle)$ approaches zero at $B=132.36 \mathrm{G}$ [Fig. 2(a)]. $\operatorname{Im}\left(a_{22}\right)$ and $\operatorname{Im}\left(a_{11}\right)$ are both negligible. To verify this prediction, we inject a probe pulse and use the slow and stopped light technique described above to transfer some state $|1\rangle$ amplitude to state $|2\rangle$ for different values of the bias magnetic field. For each field value, the number of atoms in $|2\rangle$ is measured as a function of time after the transfer, and the data are then fitted to an exponential decay. The resulting decay time, $\tau$, as a function of bias magnetic field is plotted in Fig. 2(b). The experimental results show that the largest decay time, $540 \pm 92 \mathrm{~ms}$, occurs at $B=132.4 \pm 0.1 \mathrm{G}$, which is in excellent agreement with the theoretical prediction. Improved experimental control of the bias magnetic field should allow an increase in the lifetime of the $\psi_{2}$ component by at least an order of magnitude.

Next we fix the bias field at $B=132.4 \mathrm{G}$, and a $3 \mu \mathrm{s}$ long probe pulse with a peak Rabi frequency of $4 \mathrm{MHz}$ is injected in the $+z$ direction. It is slowed to $10 \mathrm{~m} / \mathrm{s}$ and we first let it propagate all the way through the condensate, which results in a transmission of $8.5 \%$ as shown in Fig. 3(a). (The coupling peak Rabi frequency is $8 \mathrm{MHz}$ and a $10 \mu \mathrm{m}$ pinhole is placed at the image plane [Fig. 1(c)] so only probe light that has propagated through the central part of the condensate is detected). At the time indicated by an arrow, the entire probe pulse has propagated just inside and is contained in the atomic cloud. For the following experiments, the coupling laser is turned off at this time, leaving the $\psi_{2}$ imprint in the cloud [Fig. 3(b)].

We calculate [19] that at $B=132.36 \mathrm{G}$, the elastic scattering rates within and between the two condensate com-

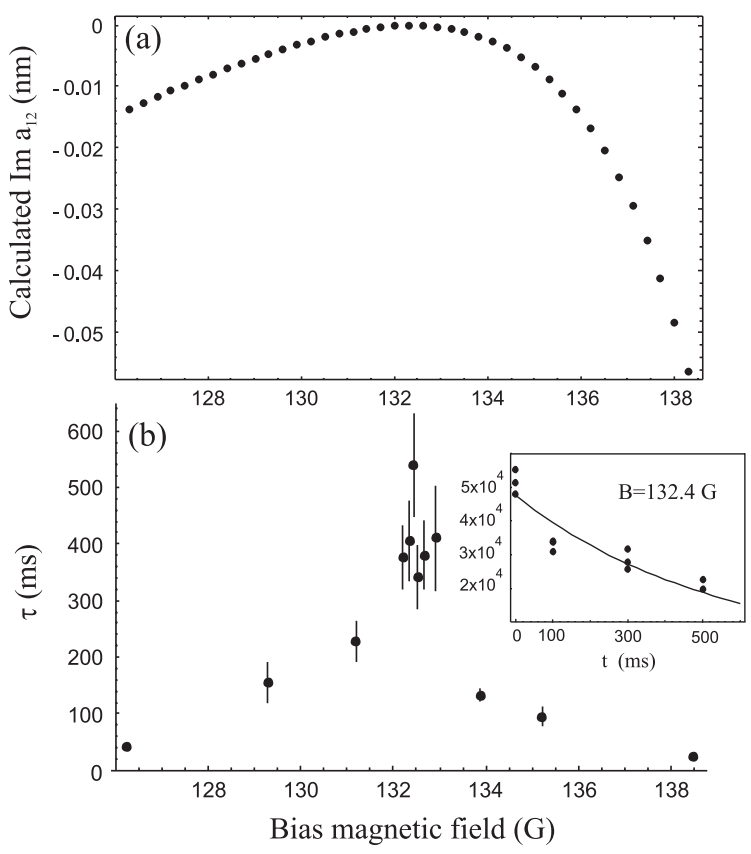

FIG. 2. (a) Calculated imaginary part of $a_{12}$ as a function of the bias magnetic field. (b) Measured decay time, $\tau$, for the atom number in the $\psi_{2}$ matter wave imprint (coexisting with the $\psi_{1}$ component) vs the bias magnetic field. The insert shows the number of $|2\rangle$ atoms as a function of time at $B=132.4 \mathrm{G}$. The data are fitted to an exponential curve with a decay time of $540 \mathrm{~ms}$. 


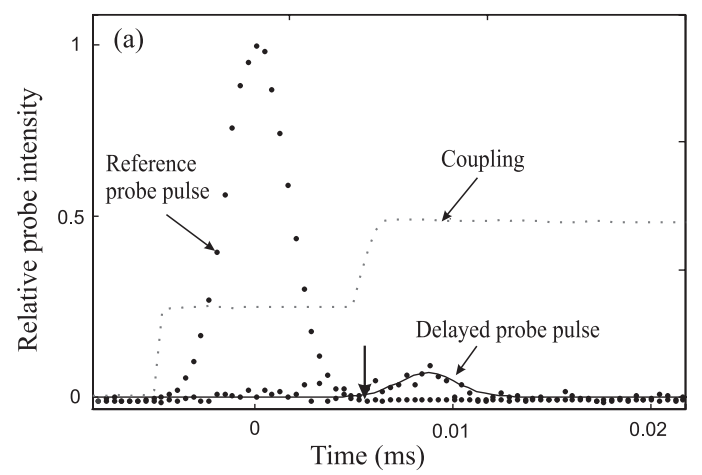

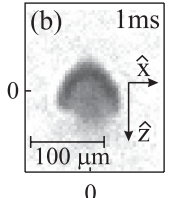

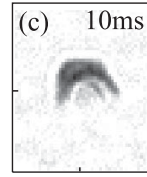

0

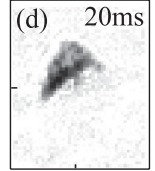

0

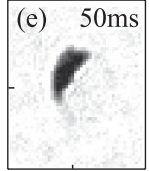

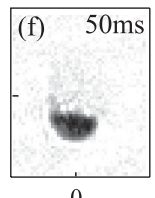

FIG. 3. (a) Probe light pulse slowed to $10 \mathrm{~m} / \mathrm{s}$ and transmitted all the way through a BEC (the reference pulse is recorded with no atoms present). (b)-(e) Images of the $\psi_{2}$ matter wave imprint at $1 \mathrm{~ms}, 10 \mathrm{~ms}, 20 \mathrm{~ms}$, and $50 \mathrm{~ms}$, respectively, after the coupling laser beam is switched off at the time indicated by an arrow in (a). (f) Same as (e) with addition of a magnetic field gradient of $200 \mathrm{mG} / \mathrm{cm}$.

ponents are determined by the atomic scattering lengths $a_{11}=2.8 \mathrm{~nm}, a_{22}=3.4 \mathrm{~nm}$, and $a_{12}=3.4 \mathrm{~nm} . \psi_{1}$ and $\psi_{2}$ are therefore in the phase-separating regime $\left(a_{11} * a_{22}<\right.$ $a_{12}^{2}$ ) [20], and Figs. 3(b)-3(e) show the observed dynamics of the $\psi_{2}$ component during the first $50 \mathrm{~ms}$ after light pulse storage. By comparing to Fig. 1(b), the $\psi_{1}$ and $\psi_{2}$ components are indeed observed to separate from each other. The $\psi_{2}$ component moves to the condensate edge, and it ends up at the upper-left corner due to a small asymmetry in the trapping potential. By adding a small magnetic gradient of $200 \mathrm{mG} / \mathrm{cm}$ along the $z$ direction, we can guide the $\psi_{2}$ component to the bottom tip of the condensate, as shown in Fig. 3(f), and at all times after $50 \mathrm{~ms}$ the $\psi_{2}$ component stably rests at this location. The inelastic loss rate of atoms is significantly lower after phase separation as shown in the insert of Fig. 2(b). In fact, if data points for the initial $50 \mathrm{~ms}$ of storage are ignored, we observe a decay time of $900 \mathrm{~ms}$ at $B=132.4 \mathrm{G}$.

When the coupling beam is turned back on after pulse storage for $50 \mathrm{~ms}$ or longer, the $\psi_{2}$ component is "shielded" behind the $\psi_{1}$ component, and where the two components overlap (roughly $10 \mu \mathrm{m}$ overlap along $z$ ), the $\psi_{2}$ component will be converted to probe light via bosonic matter wave stimulation [16]. Interestingly, we find that even that part of $\psi_{2}$ that is outside the interphase region (and therefore not overlapping with $\psi_{1}$ ) can be converted to probe light via a resonant STIRAP [21] process. The probe light revived in the interphase region propagates together with the coupling laser field into the region with a pure $\psi_{2}$ component where they act as the anti-Stokes and pump fields, respectively, in the STIRAP process. The number of photons in the output probe pulse corresponds to the number of atoms in the $\psi_{2}$ component before revival [22].

Figure 4 shows measured probe light pulses regenerated after different storage times (in this case we use a $20 \mu \mathrm{m}$ pinhole). As shown in Fig. 4(h), even after a storage time of $1.5 \mathrm{~s}$ the regenerated probe pulse is clearly observed. The revival fidelity after $1.5 \mathrm{~s}$ is $0.5 \%$ (the ratio of energy in the regenerated light pulse relative to that of the input pulse before it is injected into the cloud), and the measured fidelity dependence on storage time is consistent with the decay time observed for $\psi_{2}$ (discussed above). The temporal width of a revived light pulse reflects the shape of $\psi_{2}$ at revival, and the wide pulse in Fig. 4(a) corresponds to a $\psi_{2}$ similar to that of Fig. 3(b). Note also that no decay is observed between Figs. 4(a) and 4(b). In Fig. 4(a), the regenerated probe pulse experiences additional loss when traveling through the atomic cloud before exit whereas in Fig. 4(b), the pulse is regenerated from a $\psi_{2}$ component that has phase separated and is placed at the tip of the condensate. In the latter case the pulse can exit immediately and with no losses.

To demonstrate that the probe light pulses are regenerated through a stimulated scattering process, we perform

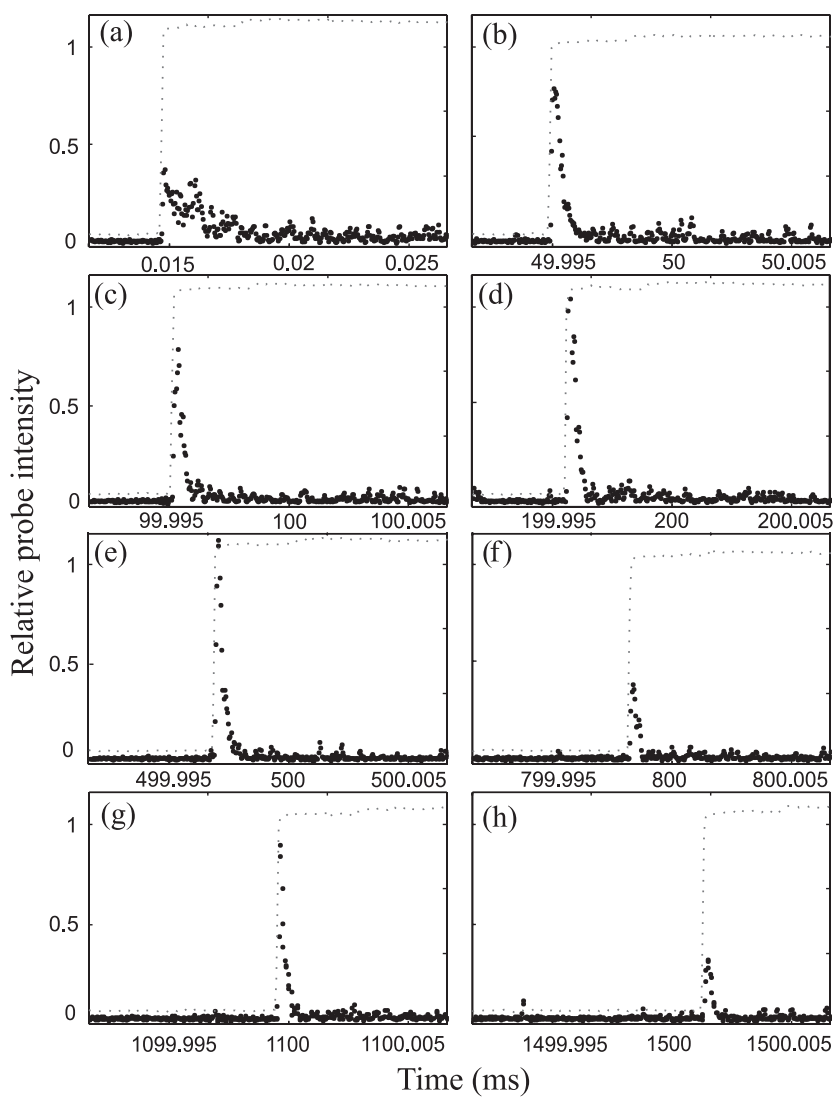

FIG. 4. Regenerated probe light pulses after storage times of $10 \mu \mathrm{s}, 50 \mathrm{~ms}, 100 \mathrm{~ms}, 200 \mathrm{~ms}, 500 \mathrm{~ms}, 800 \mathrm{~ms}, 1.1 \mathrm{~s}$, and $1.5 \mathrm{~s}$, respectively. Dashed curves indicate the intensity of the coupling beam that has a peak Rabi frequency of $12 \mathrm{MHz}$. 


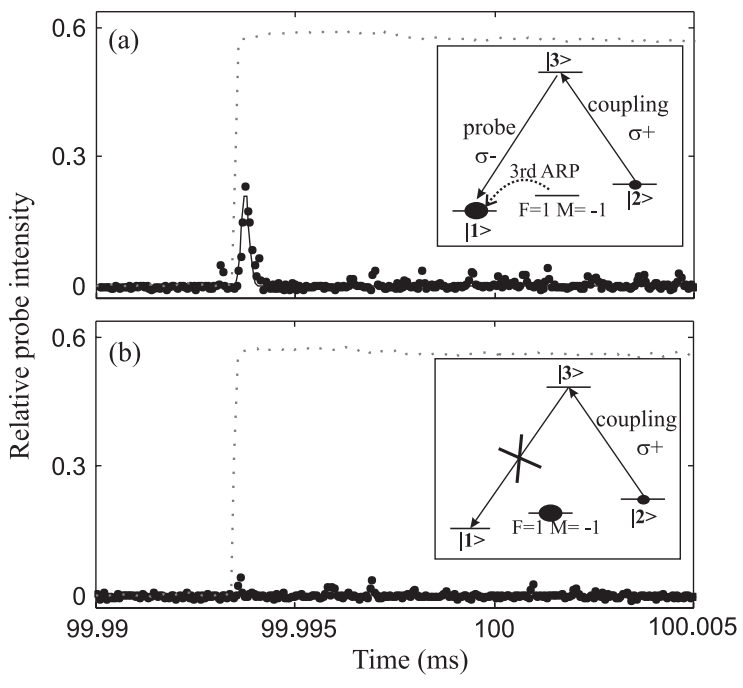

FIG. 5. (a) Regenerated probe light pulse is detected with inclusion of a third ARP step which creates the 3-level system necessary for generation of the probe field via stimulated matter wave scattering. (b) Without the third ARP step, no probe pulse is regenerated.

the following control experiment. A probe pulse is stored with the same procedure as described above except we change the magnetic field to $B=18 \mathrm{G}$. Since the $\psi_{1}$ and $\psi_{2}$ components can coexist only for about $5 \mathrm{~ms}$ at this field, a second and reverse ARP is applied to transfer atomic amplitude from $|1\rangle$ back to $|3 S, F=1, M=-1\rangle$ that can coexist with $|2\rangle$ over extended time scales. Elastic scattering lengths for this new system are similar to those for $|1\rangle$ and $|2\rangle$ at $B=132.4 \mathrm{G}$, and the dynamics of the $\psi_{2}$ component during storage is similar in the two cases. Right before the coupling beam is switched back on to regenerate the probe field, a third ARP transfers atomic amplitude from $|3 S, F=1, M=-1\rangle$ to $|1\rangle$. The probe pulse can then be successfully read out after a $100 \mathrm{~ms}$ storage time as shown in Fig. 5(a). If the third ARP step is not applied, no probe pulse is detected [Fig. 5(b)]. In this case a condensate of $|1\rangle$ atoms $\left(\psi_{1}\right)$ is not present and when the coupling laser is turned back on, amplitude in $|2\rangle\left(\psi_{2}\right)$ cannot be transferred to $|1\rangle$ by stimulated matter wave scattering [16]. (We expect that amplitude is transferred from $|2\rangle$ to $|3 S, F=1, M=-1\rangle$ with coherent generation of a linearly polarized light field traveling perpendicularly to the $z$ axis but this remains to be explored.)

In summary, we have demonstrated that light pulses can be stored in BECs for more than $1 \mathrm{~s}$. This is achieved by phase separating the atomic imprint, formed by a stored light pulse, from the main condensate and minimizing inelastic scattering. The methods applied here should work equally well for quantum states of light and could form the basis for entanglement distribution over large distances $[10,11]$. Our observations further indicate that large effective optical nonlinearities can be generated between two light pulses by utilizing the strong interactions between the associated matter imprints. With input light pulses transversely localized well below the $80 \mu \mathrm{m}$ diameter of the BEC, a rapid phase separation would ensue, leading to formation of localized voids in $\psi_{1}$ filled by the $\psi_{2}$ component. Motion of and collisions between these filled voids would be controlled with use of local magnetic field gradients. An order of magnitude increase in the revival fidelity can be achieved by such transverse localization of the input pulse, inversion of the magnetic gradient and revival of the probe pulse in the opposite $(-z)$ direction, and improved stability of the bias magnetic field. Our calculations further show that classical light pulses more intense than those used in the experiments reported here create imprints that split into two symmetric parts during phase separation. The strong atom-atom interactions, responsible for the separation, would also favor an equal number of $|2\rangle$ atoms in the two parts [23] and this should lead to spatially separated and entangled $\psi_{2}$ modes. One or both of these modes could be converted to probe light thereby generating entangled states between two separated optical modes or between the remaining $\psi_{2}$ matter wave and the revived optical pulse.

This work was supported by the Air Force Office of Sponsored Research and the National Science Foundation.

[1] H. J. Kimble, Nature (London) 453, 1023 (2008).

[2] L. V. Hau et al., Nature (London) 397, 594 (1999).

[3] C. Liu et al., Nature (London) 409, 490 (2001).

[4] D. F. Phillips et al., Phys. Rev. Lett. 86, 783 (2001).

[5] T. Chaneliere et al., Nature (London) 438, 833 (2005).

[6] M. D. Eisaman et al., Nature (London) 438, 837 (2005).

[7] K. S. Choi et al., Nature (London) 452, 67 (2008).

[8] Bo Zhao et al., Nature Phys. 5, 95 (2009).

[9] R. Zhao et al., Nature Phys. 5, 100 (2009).

[10] H.-J. Briegel et al., Phys. Rev. Lett. 81, 5932 (1998).

[11] L.-M. Duan, Phys. Rev. Lett. 88, 170402 (2002).

[12] A. V. Turukhin et al., Phys. Rev. Lett. 88, 023602 (2001).

[13] J. J. Longdell et al., Phys. Rev. Lett. 95, 063601 (2005).

[14] U. Schnorrberger et al., Phys. Rev. Lett. 103, 033003 (2009).

[15] M. Johnsson and K. Molmer, Phys. Rev. A 70, 032320 (2004).

[16] Naomi S. Ginsberg, Sean R. Garner, and Lene Vestergaard Hau, Nature (London) 445, 623 (2007).

[17] A. Ashkin, Phys. Rev. Lett. 40, 729 (1978).

[18] A. Abragam, The Principles of Nuclear Magnetism (Oxford University Press, London, 1961).

[19] J. P. Burke, Jr., C. H. Greene, and J. L. Bohn, Phys. Rev. Lett. 81, 3355 (1998).

[20] E. Timmermans, Phys. Rev. Lett. 81, 5718 (1998).

[21] K. Bergmann, H. Theuer, and B. W. Shore, Rev. Mod. Phys. 70, 1003 (1998).

[22] Detailed experimental and theoretical studies of this process will be submitted for publication.

[23] Juha Javanainen and Misha Yu. Ivanov, Phys. Rev. A 60, 2351 (1999). 